\title{
LRS: A Positive and Negative Linear Frequency Modulation Method for Ranging and Speed Measurement
}

Caixia Song ( $\nabla$ cassiesong@qau.edu.cn )

Qingdao Agriculture University: Qingdao Agricultural University https://orcid.org/0000-0003-3897-7629

Tiantian Jing

Qingdao Harbour Vocational and Technical College

\section{Research Article}

Keywords: Positive and Negative Frequency Modulation, Doppler Invariance, Distance Measurement, Speed Measurement.

Posted Date: May 7th, 2021

DOI: https://doi.org/10.21203/rs.3.rs-401335/v1

License: (c) (i) This work is licensed under a Creative Commons Attribution 4.0 International License.

Read Full License 


\title{
LRS: A Positive and Negative Linear Frequency Modulation Method for Ranging and Speed Measurement
}

\author{
Caixia Song ${ }^{*}$ and Tiantian Jing $^{2}$
}

\author{
${ }^{*}$ Correspondence: \\ cassiesong@qau.edu.cn \\ ${ }^{1}$ College of Science and \\ Information, Qingdao Agricultural \\ University, Qingdao, China \\ Full list of author information is \\ available at the end of the article
}

\begin{abstract}
Linear Frequency Modulation (LFM) is widely used in radar and sonar scenarios. However, the Doppler tolerance of LFM signal is not ideal. When the unidirectionally modulated LFM signal is in distance measurement, the Doppler delay of the matched filter output cannot be eliminated, and thus there is a ranging error. After the positive and negative Frequency Modulation (FM) echo signals, which is based on the same frequency band, are matched and filtered, the delay caused by Doppler is the same and the direction is opposite. By using the inverse time delay difference of the positive and negative FM, speed can be measured, and the ranging error in the ranging of unidirectionally modulated LFM signal can also be eliminated. In this paper, based on the analysis of the influence of target moving speed on LFM signal ranging, a positive and negative Linear frequency modulation method for Ranging and Speed measurement (LRS) is proposed. Extensive simulation results show that the proposed LRS method can better estimate the distance and speed of moving targets, and it has reference value for engineering application.
\end{abstract}

Keywords: Positive and Negative Frequency Modulation; Doppler Invariance; Distance Measurement; Speed Measurement.

\section{Introduction}

Before the occurrence of pulse compression, the distance measurement and resolution are a pair of irreconcilable contradictions in the waveform design, and the two can only be compromised. The appearance of pulse compression can solve this problem. In a large number of pulse compression signals, Linear Frequency Modulation (LFM) signal is particularly popular for good pulse compression performance. LFM contains the following advantages:

1) LFM has a certain Doppler tolerance [1], which is suitable for low-speed target detection.

2) LFM is an equal-amplitude signal, which is beneficial to improve the transmission efficiency of peak power limited systems [2].

3) A higher range resolution can be obtained by increasing the bandwidth of LFM.

4) In addition, the generation and processing techniques of LFM are relatively mature [3].

According to the above advantages, LFM has been widely used in radar and sonar. However, since the fuzzy function of the LFM signal is a oblique blade type, when the target speed is too high, Doppler mismatch is likely to occur. On the other hand, 
the target with high speed can lead to the time delay of matched filtering. This is because that the distance between the radar(sonar) and the target is determined based on the peak time of the detector's output. And thus there is a ranging error and the ranging accuracy will be reduced.

After the positive and negative Frequency Modulation (FM) echo signals, which is based on the same frequency band, are matched and filtered, the delay caused by Doppler is the same and the direction is opposite. Based on the above characteristics, a positive and negative Linear frequency modulation method for Ranging and Speed measurement (LRS) is proposed. When the LRS method is employed to measure speed, the ranging error caused by a single FM can be greatly decreased.

\section{Related work}

Whyland et al. [4] Proposed that Doppler insensitive waveforms should be used to modulate pulses or sub-pulses of energy for probing a determined environment. Therefore, when the modulated energy is transmitted and received, the received energy may be processed. X. Song et al. [5] modeled the range bias as a function of range rate and system parameters, and then utilized it to calibrate the measurement equation in very precise target tracking. By doing so, the tracking performance can be improved, particularly for fast maneuvering targets. Y. Doisy et al. [6] derived the expressions of Doppler tolerance, Doppler and delay accuracy, and delay-Doppler ambiguity in case of high bandwidth duration product signals. The replicas of Doppler estimation and target range were reduced. Finally, results were applied to low-frequency active sonar. J. Yang et al. [7] proposed a new polyphase pulse compression codes which were conceptually derived from the step approximation of the phase curve of the hyperbolic frequency modulated chirp signal. The main disadvantage of this polyphase code was the relatively high sidelobe level without Doppler effect, which can be addressed by applying the proper window function. J. Yang et al. [8] demonstrated that the acceleration of the target results in a frequency shift which is the source of the signal distortion under the assumption that the acceleration is constant and along the direction of the velocity. Therefore the frequency-shifted version of the matched filter can be applied to eliminate the mismatch between the reflected signal and the matched filter caused by the acceleration of the target. S. V. Maric et al. [9] addressed the problem of constructing frequency hop codes for use in multiuser communication systems such as multiple-access spread-spectrum communications and multiuser radar and sonar systems. The construction of a new family of frequency hopping codes called hyperbolic frequency hop codes was given, and it was shown that the hyperbolic frequency hop codes possessed nearly ideal characteristics for use in both types of system.

J. J. Kroszczynski [10] deal with the problem of wide-band signal optimization for the purpose of minimizing signal degradation resulting from Doppler distortion effects. The equation for the instantaneous frequency of a Doppler-transformed signal was derived. The optimum frequency-modulation law was then shown to be the linear period modulation. Diamant et al. [11] presented a method for Doppler-shift estimation based on comparing the arrival times of two chirp signals and approximating the relation between this time difference and the Doppler shift ratio. This analysis also provides an interesting insight about the resilience of chirp signals to 
Doppler shift. The simulation results demonstrate improvement compared to commonly used benchmark methods in terms of accuracy of the Doppler shift estimation at near-Nyquist baseband sampling rates. LFM signals have been widely used for target detection in active sonar systems due to their robustness to reverberation. D. Lee et al. [12] proposed a new fast target detection method that was robust to the variation of unknown target speed. The proposed method secured a Signal-to-Noise Ratio (SNR), approaching that of the optimal matched filter output, that was also robust to the variation of target speed and thus it was very useful for the practical use in antitorpedo torpedoes or supercavitating underwater missiles that need to equip low-complexity and robust signal processing systems. Grimmett et al. [13] proposed a method that involved using an echo-ranging system to transmit a continuously repeating linear frequency modulated signal through a propagation medium and receive a return signal reflected off of a target, performing signal processing on the return signal, extracting detected echo sets from the processing intervals, estimating a target range-rate using the estimated time versus delay slopes, computing a bias error using the estimated target range-rate, applying timing correction to the detected echo sets to remove the bias error. Guan, Chengyu et al. [14] focused on constructing an optimization model to optimize the LFM-Costas and GSFM pulse trains with the genetic algorithm. The pulse trains can be improved on properties of both ambiguity function and correlations between sub-pulses. The optimized pulse trains were proven to have better detection performance than those of the initial pulse trains. Moreover, it was affirmed that the reverberation suppression performance of pulse trains had also been improved through the optimization model. Huang, Shuxia et al. [15] proposed an effective iterative method for parameter estimation of multipath echo. The proposed method retrieves a signal component by searching for the optimal scale factor from the resampling matching outputs, and eliminates the matching output of the estimated signal to analyze the next component. The method prevented the time-domain subtractions of the received signals for providing a higher robustness. Therefore, it can be applied in many fields including active target detection and underwater communication. B. Boudamouz et al. [16] presented through the wall radar detection simulations with an emerging radar architecture which was the multiple-input multiple-output radar.

\section{Method}

3.1 Linear Frequency Modulation (LFM) signal waveform

Let $T, f_{0}, f_{1}$ and $s(t)$ represent the pulse width of the LFM signal, the starting frequency of the LFM signal, the ending frequency of the LFM signal and the LFM signal changing over time, respectively. Then, the $s(t)$ can be expressed as follows:

$$
s(t)=\left\{\begin{array}{cc}
e^{-j 2 \pi\left(f_{0} t+\frac{1}{2} \mu t^{2}\right)} \quad 0 \leq t \leq T \\
0 \quad \text { otherwise }
\end{array}\right.
$$

where $\mu=\frac{B}{T}, B=f_{1}-f_{0}$.

When $f_{1}>f_{0}$, the LFM signal is called positive LFM, denoted as $\mathrm{LFM}^{+}$. When $f_{0}>f_{1}$, the LFM signal is called negative LFM, denoted as $\mathrm{LFM}^{-}$. 


\subsection{Ranging and speed measurement principle}

For convenience, Table 1 summarizes the main symbols together with their significances in this paper.

Table 1 Summary of important symbols.

\begin{tabular}{c|l}
\hline Symbol & Definition \\
\hline$T$ & The pulse width of the LFM signal. \\
$f_{0}$ & The starting frequency of the LFM signal. \\
$f_{1}$ & The ending frequency of the LFM signal. \\
$s(t)$ & The LFM signal changing over time. \\
$v$ & The speed of the target. \\
$c$ & The speed of sound in water. \\
$R$ & The distance between the sonar transmitting point and the target. \\
$\mu(t)$ & The waveform of a single frequency signal. \\
$\mu^{*}(\mathrm{t}+\tau)$ & The conjugate of $\mu(\mathrm{t})$. \\
$\xi$ & The radial relative Doppler frequency between two targets. \\
$\tau$ & The relative delay time between two targets. \\
$\chi(\tau, \xi)$ & The fuzzy function of the signal. \\
$t_{1}$ & The time when the matched filtering maximum appears in LFM ${ }^{+}$. \\
$t_{2}$ & The time when the matched filtering maximum appears in LFM \\
$t_{a}$ & The arrival time of the pulse. \\
$\lambda$ & The signal wavelength. \\
$N_{0}$ & The noise energy. \\
$E$ & The signal energy. \\
$v_{e r r}$ & The speed measurement error of LRS method. \\
\hline
\end{tabular}

\subsubsection{The effect of speed on the echo of LFM signal}

1) The effect of the moving speed of the target on the spectrum of the $\mathrm{LFM}^{+}$signal itself

The relative movement between the sonar and the target will cause the received signal to change, which is represented by an offset in signal frequency, and such phenomenon is called the Doppler shift phenomenon [17]. Let $v$ represent the speed of the target. As can be observed in the Figure 1, when the target moves toward the sonar system, $v$ is positive and the echo frequency increases; Otherwise, $v$ is negative and the echo frequency decreases.

2) The effect of the moving speed and direction of the target on the matched filtering of $\mathrm{LFM}^{+}$signal

The matching processing gain of $10 \log (\mathrm{BT})$ can be obtained by using broadband FM signal and echo matched filtering. It can be seen from Figure 2, with the increase of the moving speed of the target, the delay caused by Doppler at the output of $\mathrm{LFM}^{+}$signal matched filtering also increases gradually. The direction of the target movement is different, and the direction of delay caused by Doppler is also different. When the sonar and the target have relative motion, the received signal will have a frequency shift, and a peak value can be obtained by using matched filtering. However, the peak value has a delay. The distance of the target is determined by the peak time of detector output, due to the delay $t_{0}$ caused by Doppler, which will reduce the measurement accuracy and lead to ranging error.

Figure 3 shows the result of matched filtering processing of positive and negative slope echo signals. It can be seen from Figure 3 that, for moving targets with positive and negative slope FM signals, the offset of delay $t_{0}$ of moving targets is the same, and the direction is just the opposite.

Note: In this paper, the positive and negative slope FM refers to: the frequency of the positive FM signal refers to the frequency rising from $f_{0}$ to $f_{1}$, and the 


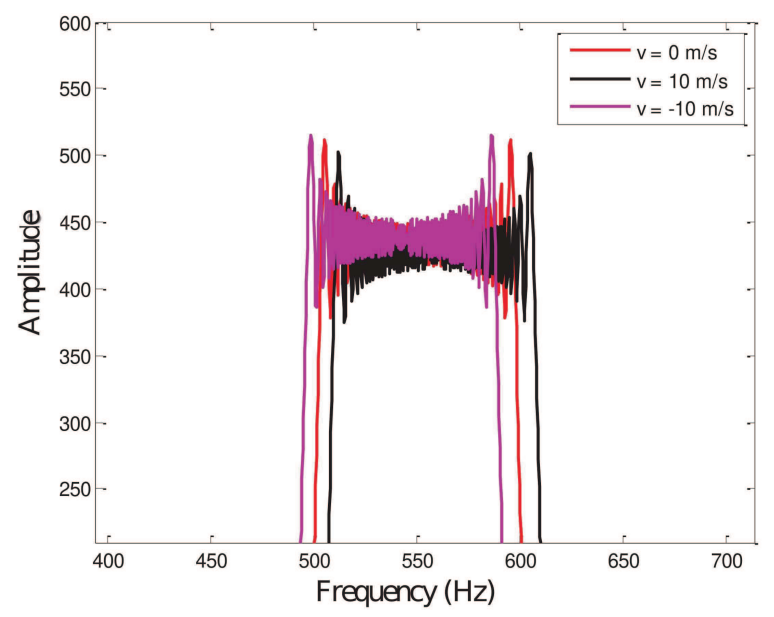

(a) The effect of velocity on Doppler

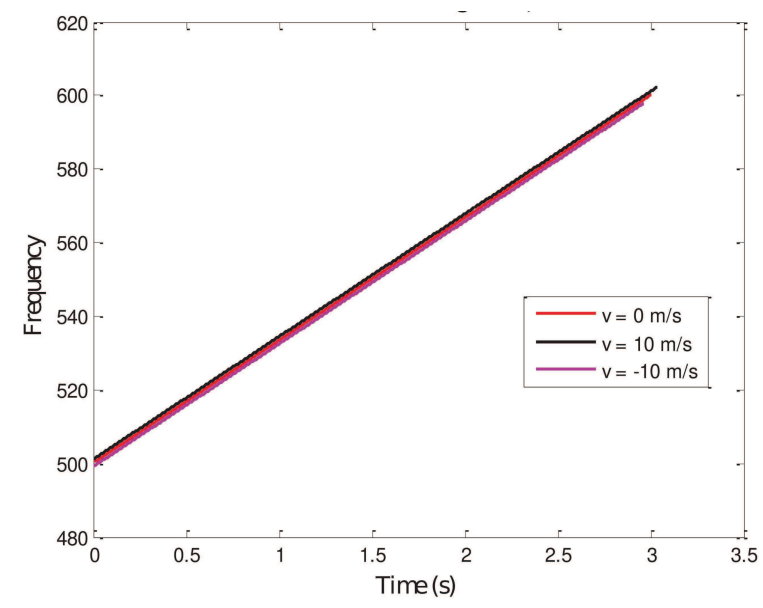

(b) LFM signal frequency varies with speed

Figure 1 The effect of target moving speed on $\mathrm{LFM}^{+}$echo spectrum

frequency of the negative FM signal refers to the frequency decreasing from $f_{1}$ to $f_{0}$. The starting and ending frequencies of the positive and negative FM signals are the same, the spectrum is the same, and the modulation slope is just different, as shown in Figure 4.

\subsubsection{Doppler characteristics of LFM singnal}

Let $\mu(t)$ represent the waveform of a single frequency signal. And then $\mu(t)$ can be expressed as:

$$
\mu(t)=\left\{\begin{array}{c}
\sqrt{1 / T} \quad 0<t<T \\
0 \quad \text { otherwise }
\end{array}\right.
$$

Let $\xi, \tau$ and $\chi(\tau, \xi)$ denote the radial relative Doppler frequency between two targets, the relative delay time between two targets and the fuzzy function of the 


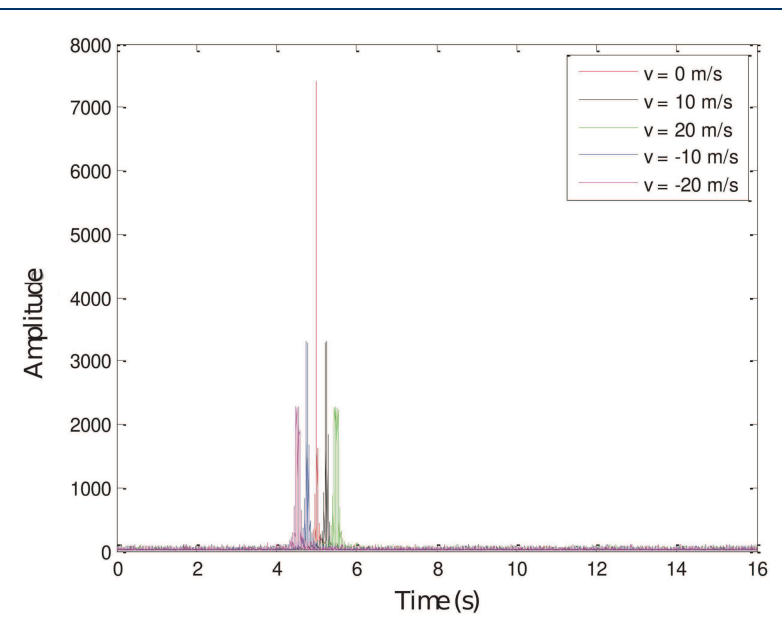

Figure 2 The effect of target's speed and direction on $\mathrm{LFM}^{+}$matched filtering signal

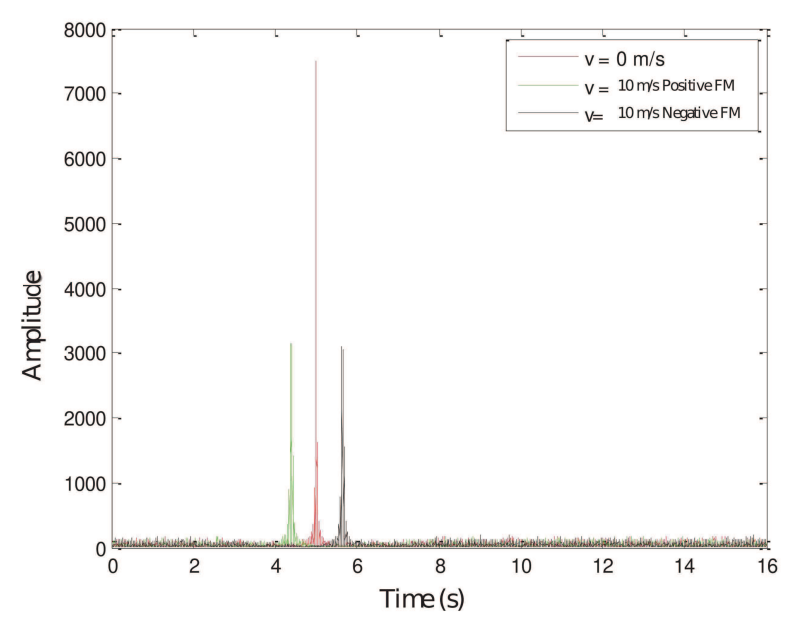

Figure 3 The delay of the target movement to the positive and negative slope FM signal

signal, respectively. And then $\chi(\tau, \xi)$ can be calculated as:

$$
\begin{aligned}
\chi(\tau, \xi) & =\int \mu(\mathrm{t}) \mu^{*}(\mathrm{t}+\tau) \mathrm{e}^{\mathrm{j} 2 \pi \xi t} d t \\
& =\int_{a}^{b} \frac{1}{T} \mathrm{e}^{\mathrm{j} 2 \pi \xi t} d t
\end{aligned}
$$

where $a$ and $b$ are the upper limits of the integral and the lower limits of the integral, respectively. And $\mu^{*}(\mathrm{t}+\tau)$ is the conjugate of $\mu(\mathrm{t})$.

The integral in the Equation (3) takes $\tau$ as the parameter, and the $\tau$ value directly affects the variation range of time $t$. Therefore, the $\frac{1}{T} \mathrm{e}^{\mathrm{j} 2 \pi \xi t}$ value in integral limit $[a, b]$ is related to the $\tau$ value. The integration process can be divided into three stages as shown in Figure 5

Stage 1: $0<\tau<T$ 


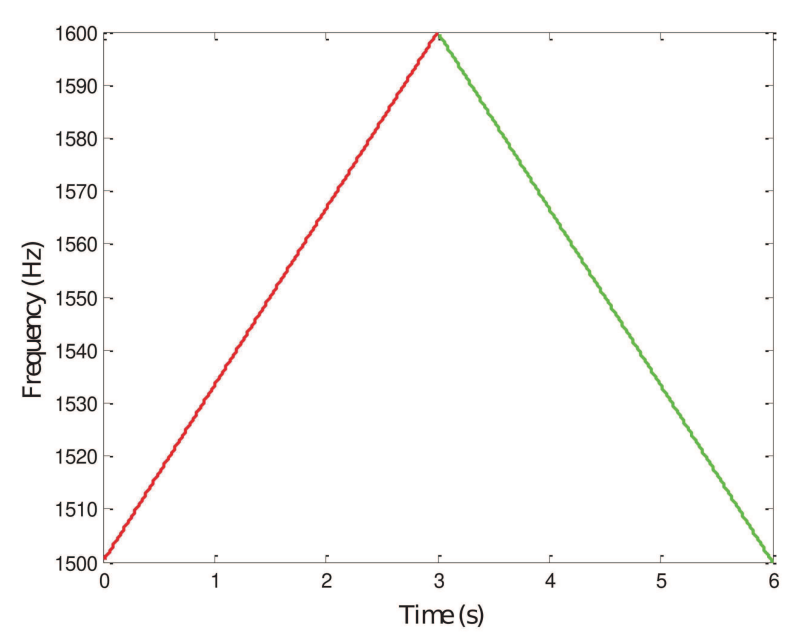

Figure 4 Schematic diagram of the spectrum of the positive and negative slope FM signals

We have $a=0, b=T-\tau$, and thus:

$$
\begin{aligned}
\chi(\tau, \xi) & =\int_{0}^{T-\tau} \frac{1}{T} e^{j 2 \pi \xi t} d t \\
& =\frac{1}{T} \frac{e^{j 2 \pi \xi t}}{j 2 \pi \xi} \mid \begin{array}{c}
T-\tau \\
0
\end{array} \\
& =\frac{1}{j 2 \pi \xi T}\left[e^{j 2 \pi \xi(T-\tau)}-1\right] \\
& =\frac{1}{T} e^{j \pi(T-\tau) \xi} \frac{e^{j \pi(T-\tau) \xi}-e^{-j \pi(T-\tau) \xi}}{j 2 \pi \xi} \\
& =e^{j \pi(T-\tau) \xi} \frac{\sin (\pi \xi(T-\tau))}{\pi \xi(T-\tau)} \frac{(T-\tau)}{T}
\end{aligned}
$$

Stage 2: $-\mathrm{T}<\tau<0$

We have $a=-\tau, b=T$, and thus:

$$
\begin{aligned}
\chi(\tau, \xi) & =\int_{-\tau}^{T} \frac{1}{T} e^{j 2 \pi \xi t} d t \\
& =\frac{1}{T} e^{-j 2 \pi \xi \tau} \frac{e^{j 2 \pi \xi(T+\tau)}-1}{j 2 \pi \xi} \\
& =e^{j \pi \xi(T-\tau)} \frac{\sin (\pi \xi(T+\tau))}{\pi \xi(T+\tau)} \frac{T+\tau}{T}
\end{aligned}
$$

Stage 3: $\tau>T$ or $\tau<-T$

Since the integral function is 0 , and thus:

$$
\chi(\tau, \xi)=0
$$




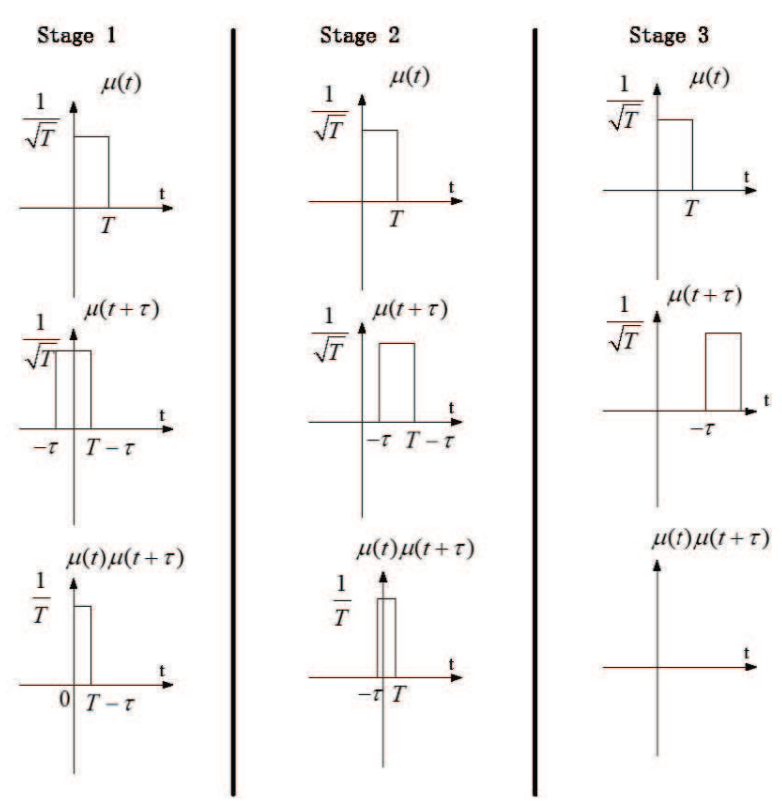

Figure 5 Three stages of the integration process.

In summary, we have:

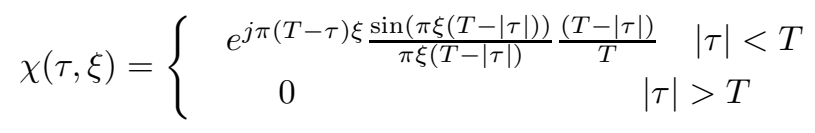

Equation (7) is also the fuzzy function of a single frequency signal. According to the transformation property of the fuzzy function [18], the fuzzy function of LFM is

$$
\chi(\tau, \xi)=\left\{\begin{array}{cc}
e^{j\left(\pi(\xi-\mu \tau)(T-\tau)-\mu \tau^{2} \pi\right) \frac{\sin \pi(\xi-\mu \tau)(T-|\tau|)}{\pi(\xi-\mu \tau)(T-\tau)}(T-|\tau|)} & |\tau|<T \\
0 & |\tau|>T
\end{array}\right.
$$

Then, we have

$$
|\chi(\tau, \xi)|=\frac{\sin \pi(\xi-\mu \tau)(T-|\tau|)}{\pi(\xi-\mu \tau)(T-|\tau|)}(T-|\tau|)
$$

For the cross section: $\xi-\mu \tau=0$, we have

$$
\chi(\tau, \xi)=T-|\tau|
$$

$\chi(\tau, \xi)$ decreases linearly as $\tau$ increases. And then the pulse pressure delay caused by the moving target is $\tau=\frac{\xi}{\mu}$.

When the sonar and the target are in relative movement, the received signal will have a frequency shift. Since LFM has a certain Doppler tolerance, the instantaneous 
frequency of the received signal only has a time delay. Therefore, a good peak can be obtained with matched filtering, but there is a time delay $t_{0}$ in the position of the peak. $t_{0}$ can be calculated as:

$$
t_{0}=\frac{f_{d}}{\frac{f_{1}-f_{0}}{T}}
$$

where $f_{d}$ can be approximated as $\frac{2 v}{\lambda}$, and $\lambda$ denotes the signal wavelength. We have

$$
\lambda=\frac{c}{\frac{f_{1}+f_{0}}{2}}
$$

where $c$ represents the speed of sound in water and $c=1500 \mathrm{~m} / \mathrm{s}$. Therefore, we have

$$
t_{0}=\frac{\frac{2 v}{c} \frac{f_{1}+f_{0}}{2}}{\frac{f_{1}-f_{0}}{T}}
$$

\subsection{Ranging and speed measurement based on positive and negative slope frequency modulation}

Now let's derive the distance measurement formula. We assume that the movement speed $v$ of the target is positive toward the sonar system. First, a $\mathrm{LFM}^{+}$signal whose frequency increases with time is transmitted, and then a $\mathrm{LFM}^{-}$signal whose frequency decreases with time is transmitted. Let $t_{1}$ and $t_{2}$ represent the time when the matched filtering maximum appears in $\mathrm{LFM}^{+}$and $\mathrm{LFM}^{-}$, respectively. We have

$$
\begin{aligned}
& t_{1}=\frac{2 R}{c}+t_{0} \\
& t_{2}=\frac{2 R}{c}-t_{0}
\end{aligned}
$$

where $R$ represents the distance between the sonar transmitting point and the target.

According to Equation (14) and Equation (15), we have

$$
\begin{aligned}
R & =\frac{\left(t_{1}+t_{2}\right) \times c}{4} \\
& =\frac{1}{2} \times c \times \frac{\left(t_{1}+t_{2}\right)}{2}
\end{aligned}
$$

The distance $R$ can also be expressed as:

$$
R=\frac{1}{2} \times c \times t_{a}
$$

where $t_{a}$ represents the arrival time of the pulse.

According to Equation (16) and Equation (17), $t_{a}$ is obtained.

$$
t_{a}=\frac{2 R}{c}=\frac{\left(t_{1}+t_{2}\right)}{2}
$$


According to Equation (13), (14) and (18), we have

$$
v=\frac{c}{\frac{f_{1}+f_{0}}{2}} \times \frac{\frac{f_{1}-f_{0}}{T}\left(t_{1}-\frac{\left(t_{1}+t_{2}\right)}{2}\right)}{2}
$$

Let $v_{\text {err }}$ denotes the speed measurement error of LRS method, $v_{\text {err }}$ can be calculated by

$$
v_{e r r}=\sqrt{\frac{3}{\frac{2 E}{N_{0}}(\pi T)^{2}}}
$$

where $N_{0}$ and $E$ denotes the noise energy and the signal energy, respectively.

The LRS is a ranging and speed measurement method, and it is based on the principle that, after the combined positive and negative FM signal are matched filtering, the moving target has opposite delay direction. Therefore, the LRS method is also suitable for radar scenarios.

\section{Results and Discussion}

\subsection{Simulation Settings}

We set up two simulation environments for performance analysis: Simulation Environment 1 and Simulation Environment 2.

Simulation Environment 1: The pulse width of LFM signal $T=3 \mathrm{~s}$ and sampling frequency $f_{s a}=5000 \mathrm{~Hz}$. The speed of the target is $10 \mathrm{~m} / \mathrm{s}$. The distance between target and sound source is $3.75 \mathrm{~km}$. In the echo signal, the SNR is $-10 \mathrm{~dB}$. The starting and ending frequency of positive and negative $\mathrm{FM}$ are $f_{0}=1500 \mathrm{~Hz}$ and $f_{1}$ $=1600 \mathrm{~Hz}$, respectively.

Simulation Environment 2: The pulse width of LFM signal $T=3 \mathrm{~s}$ and sampling frequency $f_{s a}=7000 \mathrm{~Hz}$. The speed of the target is $20 \mathrm{~m} / \mathrm{s}$. The distance between target and sound source is $15 \mathrm{~km}$. In the echo signal, the SNR is $-20 \mathrm{~dB}$. The starting and ending frequency of positive and negative FM are $f_{0}=3000 \mathrm{~Hz}, f_{1}=3100 \mathrm{~Hz}$, respectively.

\subsection{Comparison with Existing Studies}

Figure 6 shows the performance analysis under the Simulation Environment 1 under various $v$. Table 2 gives the numerical results of LRS, $\mathrm{LFM}^{+}$and $\mathrm{LFM}^{-}$.

We take Figure 6(a) as an example to analyze the performance of LRS method under various speed $v$. From Figure $6(\mathrm{a})$, it can be seen that, in the LRS method, after matched filtering, the maximum points $t_{1}=4.403$ and $t_{2}=5.637$. Based on Equation (16), $R$ is equal to $3.7650 \mathrm{~km}$. According to Equation (19), the value of $v$ is $9.9516 \mathrm{~m} / \mathrm{s}$. The speed measurement error and the ranging error of LRS are $0.4840 \%$ and $0.4000 \%$, respectively. The ranging error of $\mathrm{LFM}^{+}$and $\mathrm{LFM}^{-}$are $11.9400 \%$ and $12.7400 \%$, respectively. It can be seen from Table 2 that, compared with $\mathrm{LFM}^{+}$ and $\mathrm{LFM}^{-}$, the range measurement accuracy of LRS is improved by $96.64992 \%$ and $96.86028 \%$, respectively.

Figure 7 shows the performance analysis under the Simulation Environment 2

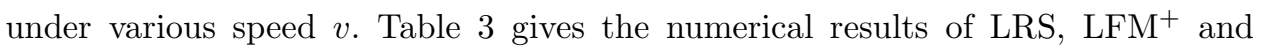
$\mathrm{LFM}^{-}$. 
Table 2 Results under Simulation Environment 1

\begin{tabular}{|c|c|c|c|c|c|c|c|c|c|c|}
\hline speed $v$ & Peak & ioment & Speed measurement & Ranging of & Speed measurement & Ranging error & Ranging er & of single signal & Range accuracy & improvement ratio \\
\hline$(\mathrm{m} / \mathrm{s})$ & & & of LRS $(\mathrm{m} / \mathrm{s})$ & $\operatorname{LRS}(\mathrm{km})$ & error of LRS & of LRS & $\mathrm{LFM}^{+}$ & LFM" $^{-1}$ & \begin{tabular}{|l|l} 
Compared to LFM+ \\
\end{tabular} & Compared to LFM- \\
\hline 10 & 4.403 & 5.637 & 9.9516 & 3.7650 & $0.4840 \%$ & $0.4000 \%$ & $11.9400 \%$ & $12.7400 \%$ & $96.64992 \%$ & $96.86028 \%$ \\
\hline-10 & \begin{tabular}{|l|l|}
4.354 \\
\end{tabular} & 5.606 & -10.0968 & 3.7350 & $0.9680 \%$ & $0.4000 \%$ & $12.92 \%$ & $12.12 \%$ & $\overline{96.90402 \%}$ & $\overline{96.69967 \%}$ \\
\hline
\end{tabular}

Table 3 Results under Simulation Environment 2

\begin{tabular}{|c|c|c|c|c|c|c|c|c|c|c|}
\hline speed $v$ & Peak r & moment & \begin{tabular}{|l|l} 
Speed $n$ \\
\end{tabular} & Ranging of & eeed $n$ & Ranging er & Ranging & g error of sing & acy & 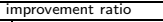 \\
\hline & & $t_{2}$ & of LRS $(\mathrm{m} / \mathrm{s})$ & LRS (km) & error of LRS & of LRS & & LFM & ompared to LFM $^{+}$ & Compared to LFM \\
\hline 20 & & 22.55 & 20.6148 & 15.0262 & & $0.1747 \%$ & & 12.75 & $98.59113 \%$ & $98.6298 \%$ \\
\hline 5 & \begin{tabular}{|l|}
19.39 \\
\end{tabular} & 20.62 & 5.0410 & 15.0038 & $0.8197 \%$ & $0.025 \%$ & $3.05 \%$ & $3.1 \%$ & $99.18033 \%$ & $99.19355 \%$ \\
\hline
\end{tabular}

We take Figure 7(a) as an example to analyze the performance of LRS method under various $v$. From Figure 7(a), it can be seen that, in the LRS method, after matched filtering, the maximum points $t_{1}=17.520$ and $t_{2}=22.55$. Based on Equation (16), $R$ is equal to $15.0262 \mathrm{~km}$. According to Equation (19), the value of $v$ is $20.6148 \mathrm{~m} / \mathrm{s}$. The speed measurement error and the ranging error of LRS are $3.0740 \%$ and $0.1747 \%$, respectively. The ranging error of $\mathrm{LFM}^{+}$and $\mathrm{LFM}^{-}$are $12.4 \%$ and $12.75 \%$, respectively. It can be seen from Table 3 that, compared with $\mathrm{LFM}^{+}$and $\mathrm{LFM}^{-}$, the range measurement accuracy of LRS is improved by $98.59113 \%$ and $98.6298 \%$, respectively. On the other hand, from Figure 7(a) and 7(b), it is clear that the smaller the moving speed of the target, the smaller of the ranging error and the speed measurement error.

\section{Conclusion}

The LFM signal can solve the contradiction between the distance measurement and range resolution, therefore, it is widely used in sonar and radar detection field. The single LFM signal's tolerance towards Doppler of moving targets is not ideal, and the target movement will induce the time delay $t_{0}$ at the output of matched filter, which will lead to ranging error. In this paper, a positive and negative Linear frequency modulation method for Ranging and Speed measurement (LRS) is proposed. The LRS method can eliminate the ranging error caused by Doppler time delay $t_{0}$ of single LFM signal, and then the accurate speed measurement is achieved.

The ideal transmission channel is an infinite space composed of lossless and uniform medium, and the signal will not have any distortion during the propagation process. However, the sea water medium space is a lossy heterogeneous medium space. In addition to general absorption and diffusion, the signal in sea water is also affected by multi-path effect, channel time-varying and fluctuation effect, resulting in the broadening of the echo and the difficulty in distinguishing the echo position of the combined echo signal. In order to achieve accurate speed measurement, the time delay $t_{0}$ should be as large as possible. According to Equation (11), to make $t_{0}$ larger, the following methods can be adopted: 1) Increase the pulse width $T$ of the detection signal. 2) Increase the signal frequency $f_{d}$. 3) Decrease the bandwidth $\left(f_{1}-f_{0}\right)$.

\section{Abbreviations}

LFM: Linear Frequency Modulation; FM: Frequency Modulation; LRS: a positive and negative Linear frequency modulation method for Ranging and Speed measurement; SNR: Signal-to-Noise Ratio.

\section{Acknowledgements}

We acknowledge the support of Yuankun Peng from Hangzhou Institute of Applied Acoustics for his critical remarks. 


\section{Authors' contributions}

CS conceived the study, conducted the experiments, supervised the study and wrote the paper. TJ reviewed and edited the manuscript. The authors read and approved the final manuscript.

\section{Funding}

This work was supported in part by the Shandong Smart Ocean Ranch Engineering Technology Collaborative Innovation Center, in part by the research fund for high-level talents of Qingdao Agricultural University (NO.1119048), in part by the Shandong Agricultural Science and Technology Service Project (NO.2019FW037-4), in part by Shandong Technology Innovation Guidance Program (NO.2020LYXZ023), in part by Horizontal Project (NO.20193702010792), in part by Experimental technical project of Qingdao Agricultural University (NO.SYJK18-01), and in part by Ministry of Education Industry-University Cooperation Collaborative Education Project (NO.201902005027 and NO.201901029013).

\section{Availability of data and materials}

Please contact the corresponding author for simulation results.

\section{Declarations}

\section{Consent for publication}

Informed consent was obtained from all authors included in the study.

\section{Competing interests}

The authors declare that they have no competing interests.

\section{Author details}

${ }^{1}$ College of Science and Information, Qingdao Agricultural University, Qingdao, China. ${ }^{2}$ School of Information and Electrical Engineering, Qingdao Harbour Vocational and Technical College, Qingdao, China.

\section{References}

1. Kim, K.-T.: Focusing of high range resolution profiles of moving targets using stepped frequency waveforms. IET radar, sonar \& navigation 4(4), 564-575 (2010)

2. Patton, L., Frost, S., Rigling, B.: Efficient design of radar waveforms for optimised detection in coloured noise. IET Radar, Sonar \& Navigation 6(1), 21-29 (2012)

3. Łuszczyk, M., Łabudzinski, A.: Sidelobe level reduction for complex radar signals with small base. In: 201213 th International Radar Symposium, pp. 146-149 (2012). IEEE

4. Whyland, W.P.: Doppler consistent hyperbolic frequency modulation. Google Patents. US Patent $5,077,702$ (1991)

5. Song, X., Willett, P., Zhou, S.: Range bias modeling for hyperbolic-frequency-modulated waveforms in target tracking. IEEE journal of Oceanic Engineering 37(4), 670-679 (2012)

6. Doisy, Y., Deruaz, L., Beerens, S.P., Been, R.: Target doppler estimation using wideband frequency modulated signals. IEEE transactions on signal processing 48(5), 1213-1224 (2000)

7. Yang, J., Sarkar, T.K.: A new doppler-tolerant polyphase pulse compression codes based on hyperbolic frequency modulation. In: 2007 IEEE Radar Conference, pp. 265-270 (2007). IEEE

8. Yang, J., Sarkar, T.K.: Acceleration-invariant pulse compression using hyperbolic frequency modulated waveforms. In: 2006 International Waveform Diversity \& Design Conference, pp. 1-5 (2006). IEEE

9. Maric, S.V., Titlebaum, E.L.: A class of frequency hop codes with nearly ideal characteristics for use in multiple-access spread-spectrum communications and radar and sonar systems. IEEE Transactions on Communications 40(9), 1442-1447 (1992)

10. Kroszczynski, J.J.: Pulse compression by means of linear-period modulation. Proceedings of the IEEE 57(7), 1260-1266 (1969)

11. Diamant, R., Feuer, A., Lampe, L.: Choosing the right signal: Doppler shift estimation for underwater acoustic signals. In: Proceedings of the Seventh ACM International Conference on Underwater Networks and Systems. WUWNet '12, pp. 27-1278. ACM, New York, NY, USA (2012). doi:10.1145/2398936.2398971. http://doi.acm.org/10.1145/2398936.2398971

12. Lee, D., Shin, J., Do, D., Choi, S., Kim, H.: Robust Ifm target detection in wideband sonar systems. IEEE Transactions on Aerospace and Electronic Systems 53(5), 2399-2412 (2017). doi:10.1109/TAES.2017.2696318

13. Grimmett, D.J.: System and method for target doppler estimation and range bias compensation using high duty cycle linear frequency modulated signals. Google Patents. US Patent 9,465,108 (2016)

14. Guan, C., Zhou, Z., Zeng, X.: Optimal waveform design using frequency-modulated pulse trains for active sonar. Sensors 19(19), $4262(2019)$

15. Huang, S., Fang, S., Han, N.: Iterative matching-based parameter estimation for time-scale underwater acoustic multipath echo. Applied Acoustics 159, 107094 (2020) 
16. Boudamouz, B., Millot, P., Pichot, C.: Through the wall mimo radar detection with stepped frequency waveforms. In: The 7th European Radar Conference, pp. 400-402 (2010). IEEE

17. Tan, T.: Sonar Technology. Harbin Engineering University Press, ??? (2010)

18. Rui, G., amd Zhi-xiang, C.Z.-m.: Sidelobe suppression method of linear frequency modulated signal without mainlobe widening. Journal of Electronics \& Information Technology 36(002), 298-303 (2014) 


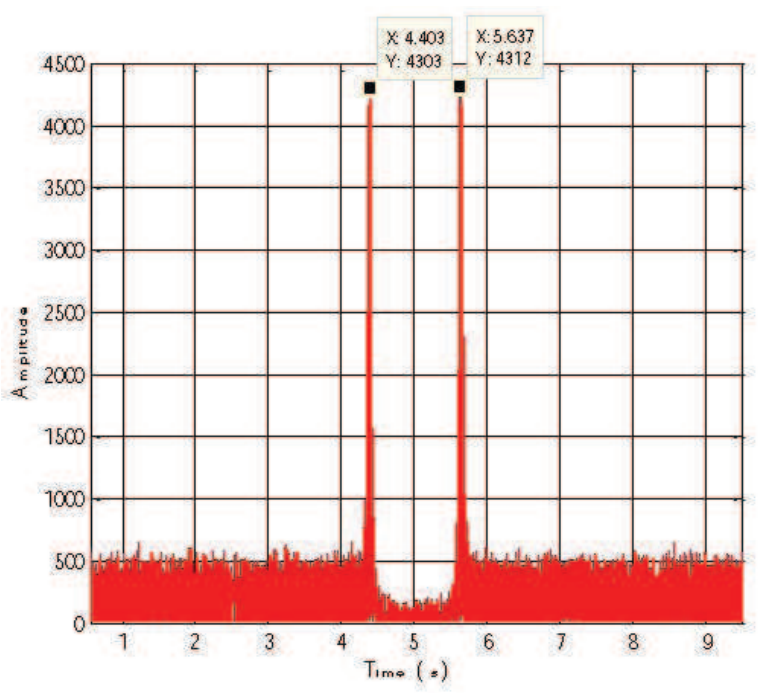

(a) $v=10 \mathrm{~m} / \mathrm{s}$

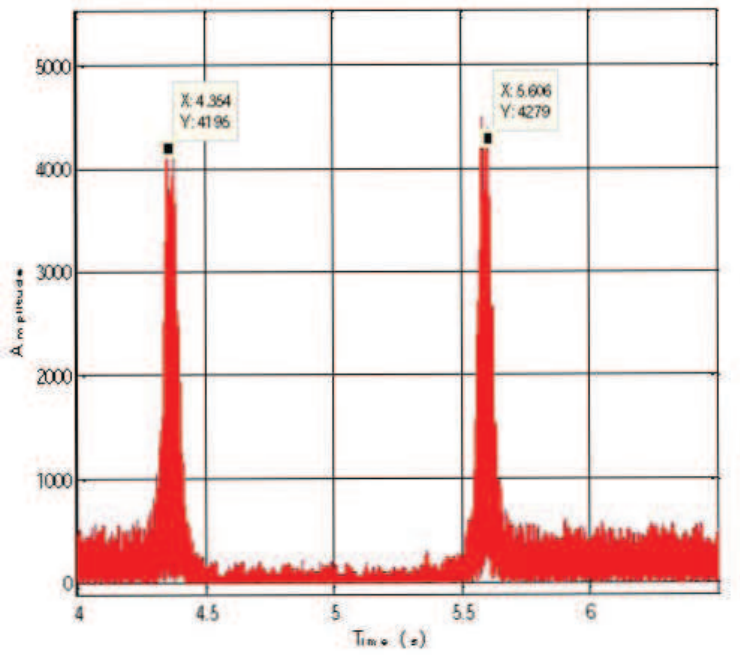

(b) $v=-10 \mathrm{~m} / \mathrm{s}$

Figure 6 The output of matched filtering under various $v$ 


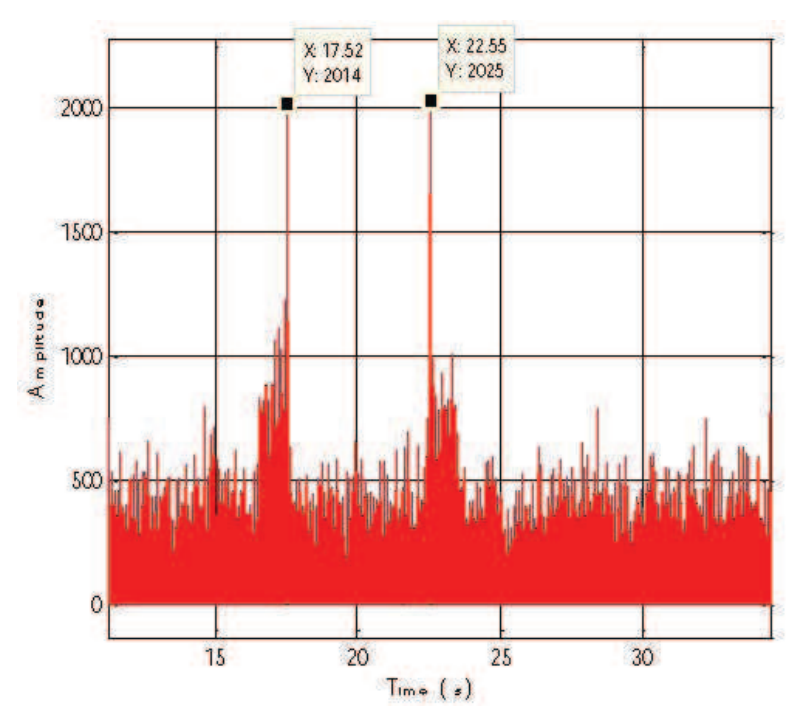

(a) $v=20 \mathrm{~m} / \mathrm{s}$

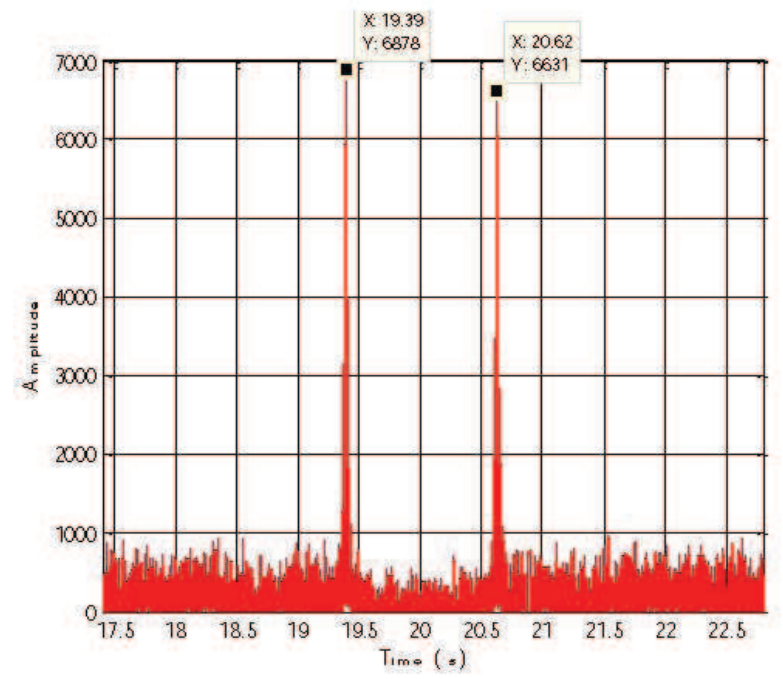

(b) $v=5 \mathrm{~m} / \mathrm{s}$

Figure 7 The output of matched filtering under various $v$ 
Figures

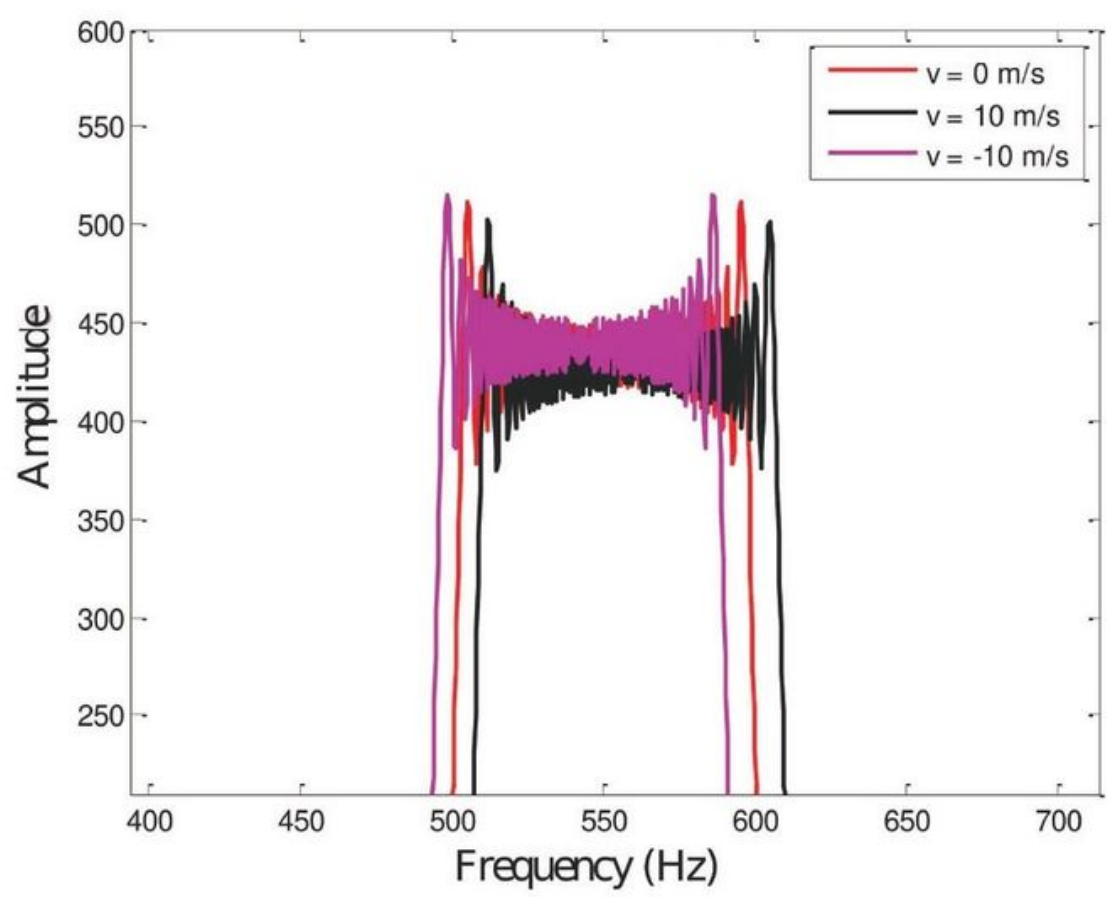

(a) The effect of velocity on Doppler

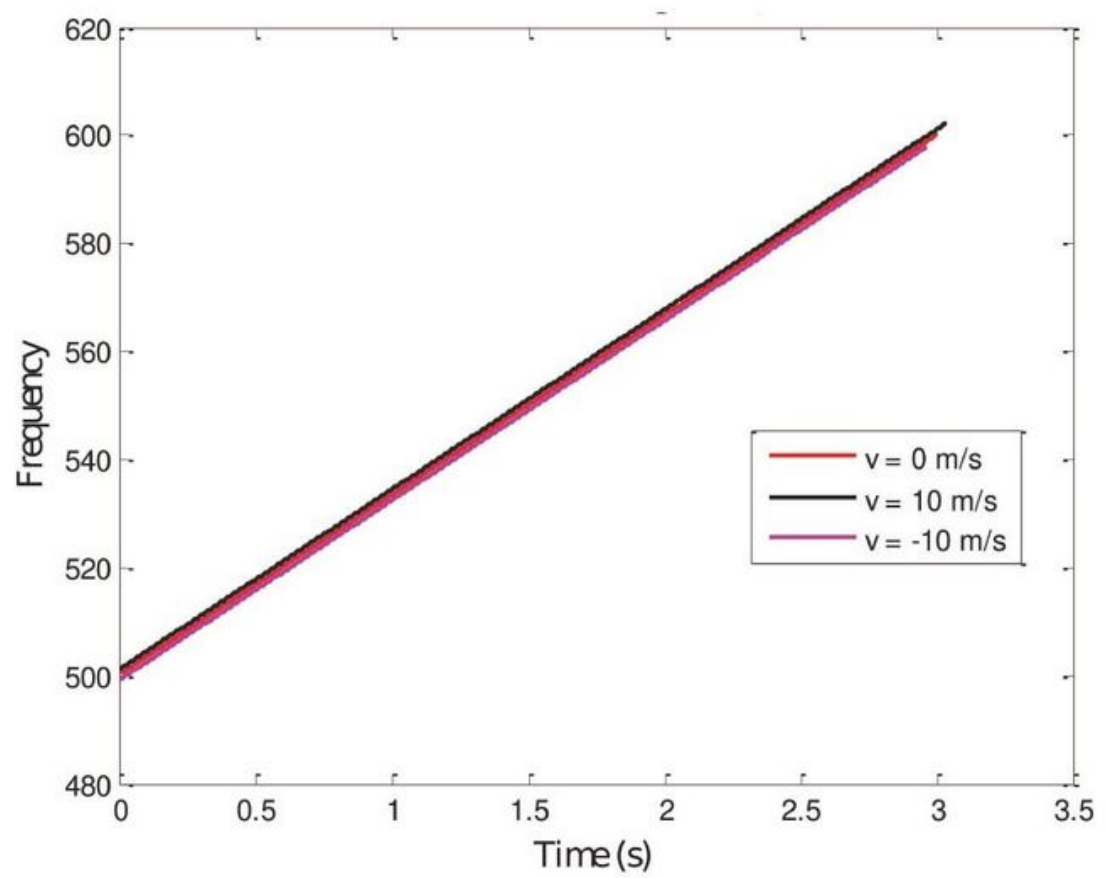

(b) LFM signal frequency varies with speed

\section{Figure 1}

The effect of target moving speed on LFM+ echo spectrum 


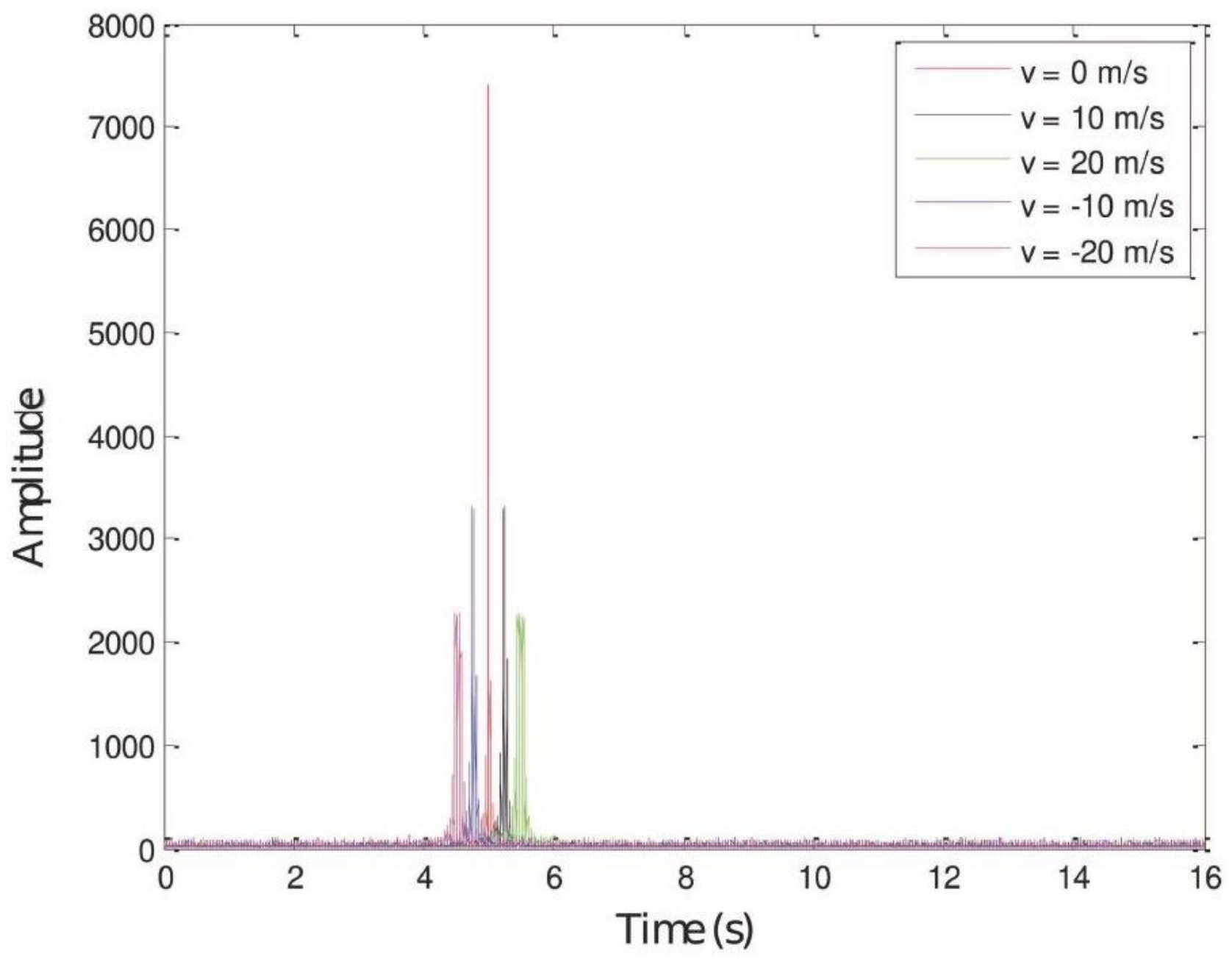

Figure 2

The effect of target's speed and direction on LFM+ matched filtering signal 


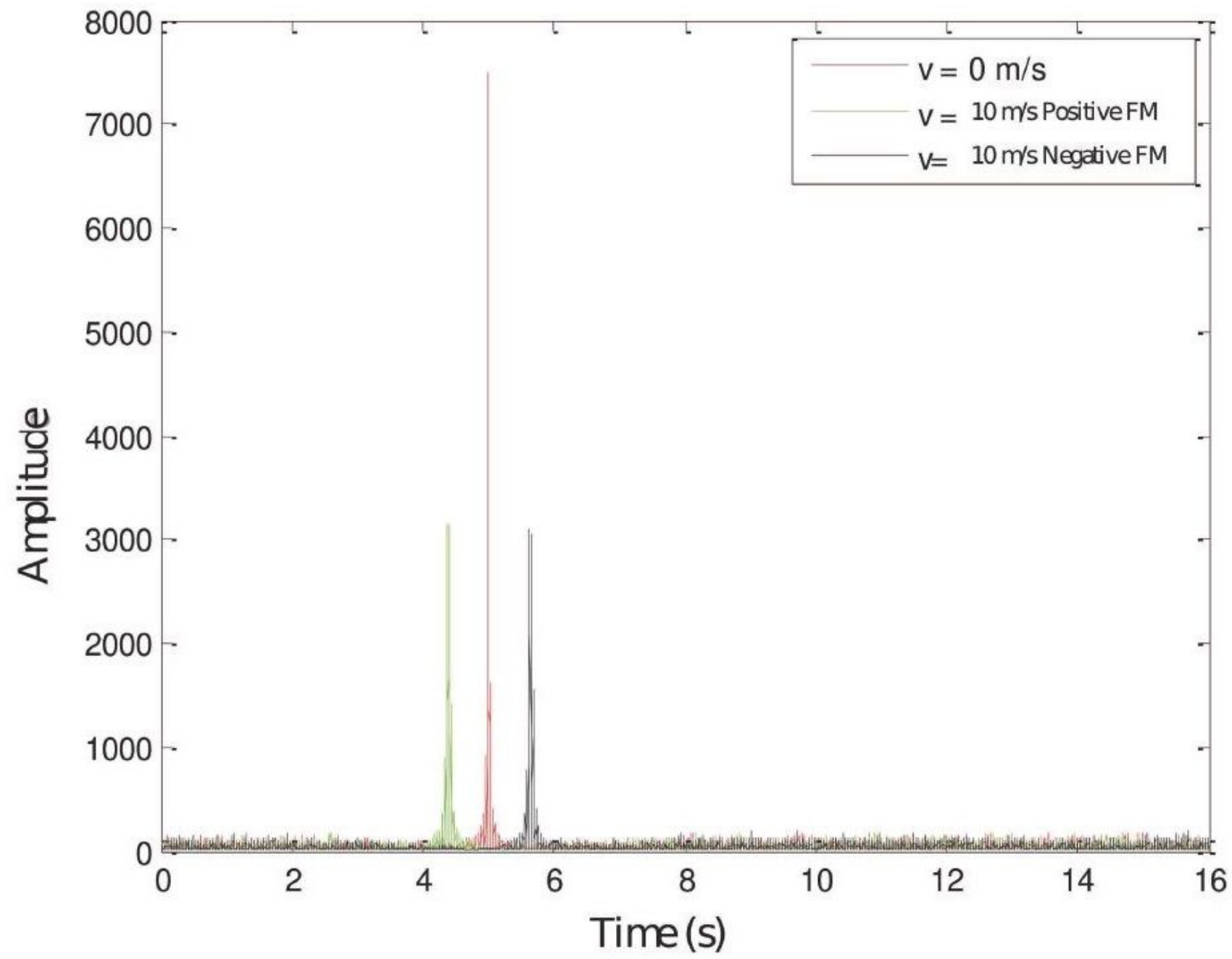

Figure 3

The delay of the target movement to the positive and negative slope FM signal 


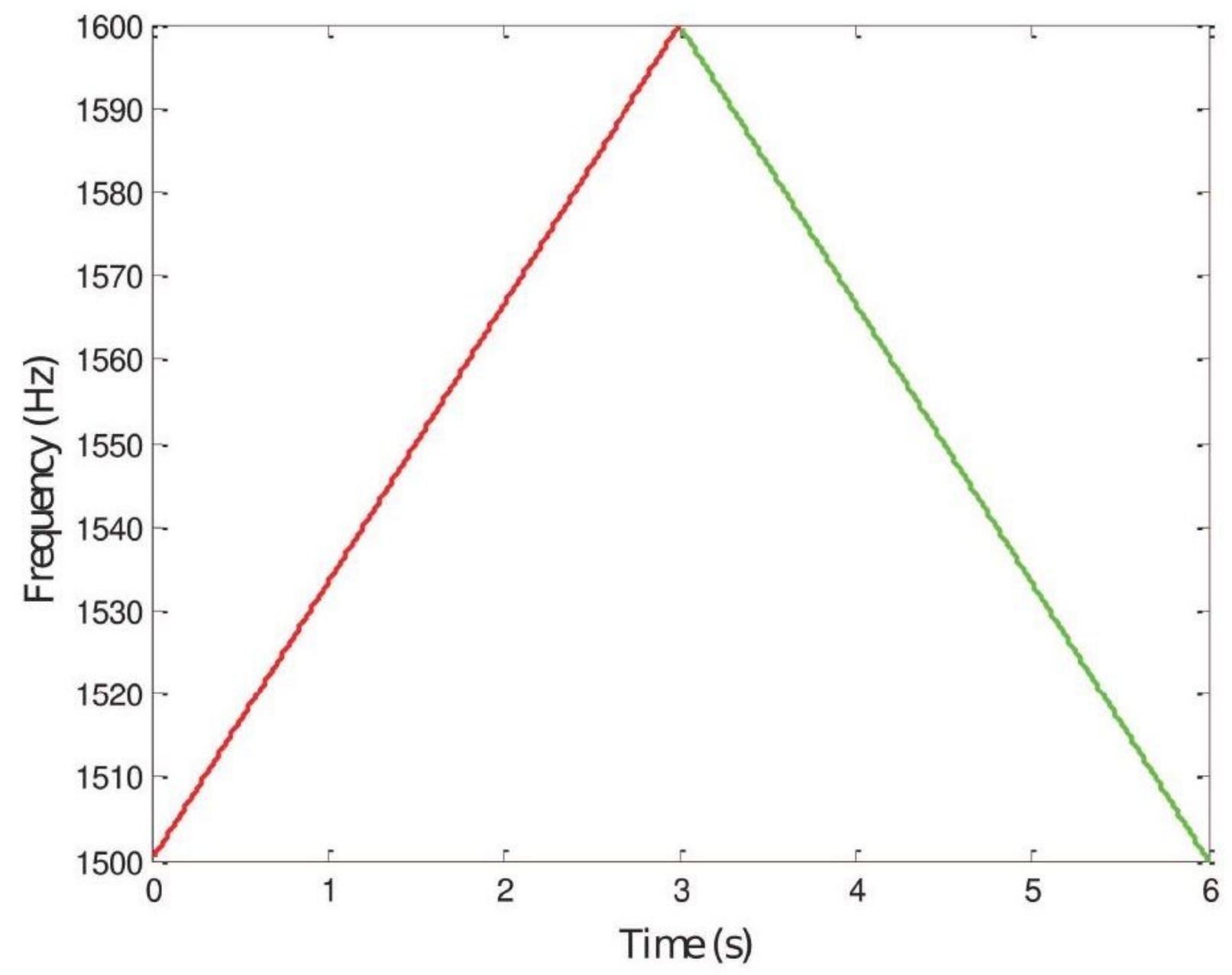

Figure 4

Schematic diagram of the spectrum of the positive and negative slope FM signals 


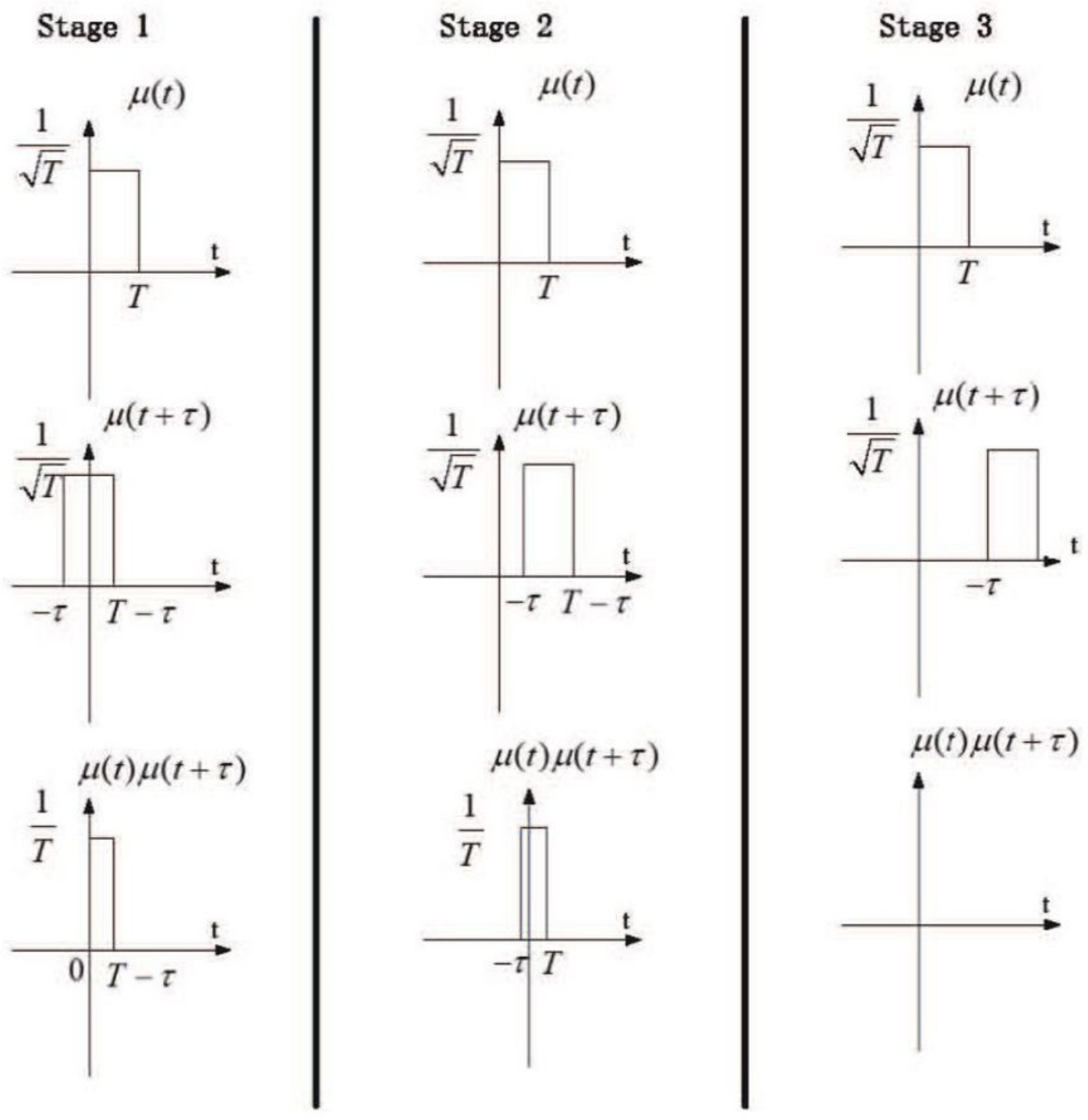

Figure 5

Three stages of the integration process. 


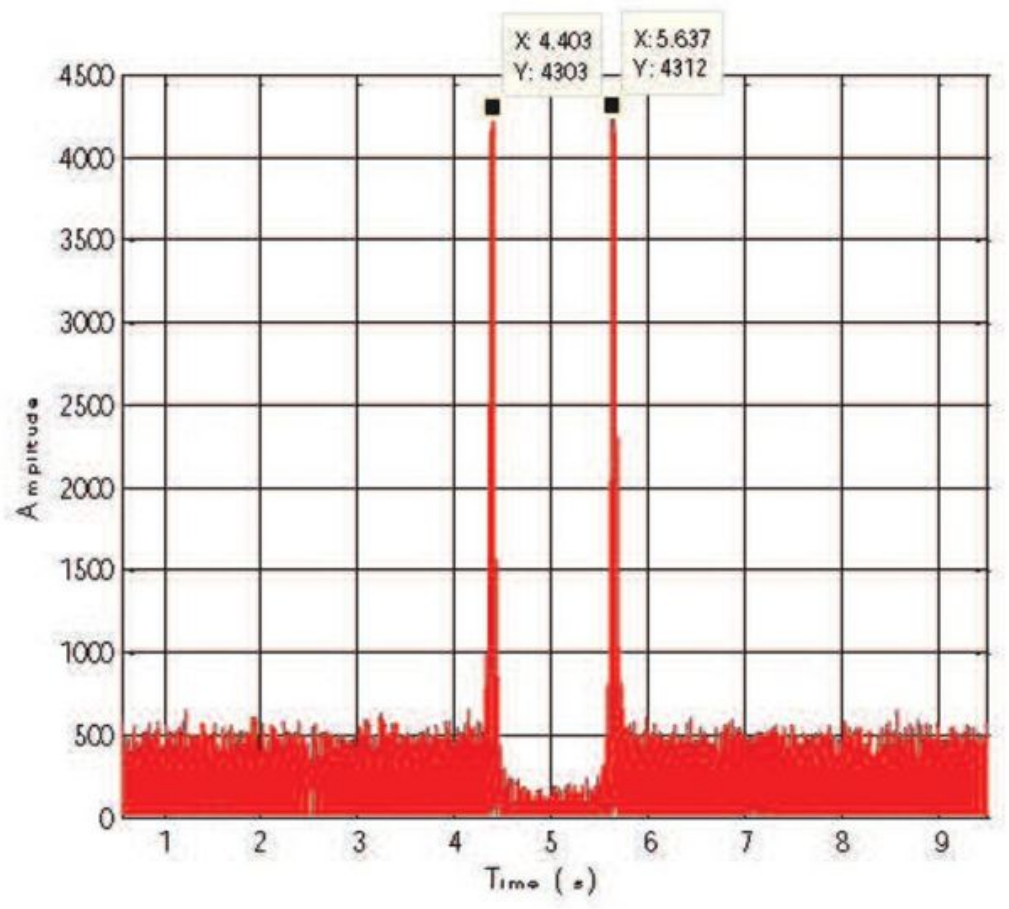

(a) $v=10 \mathrm{~m} / \mathrm{s}$

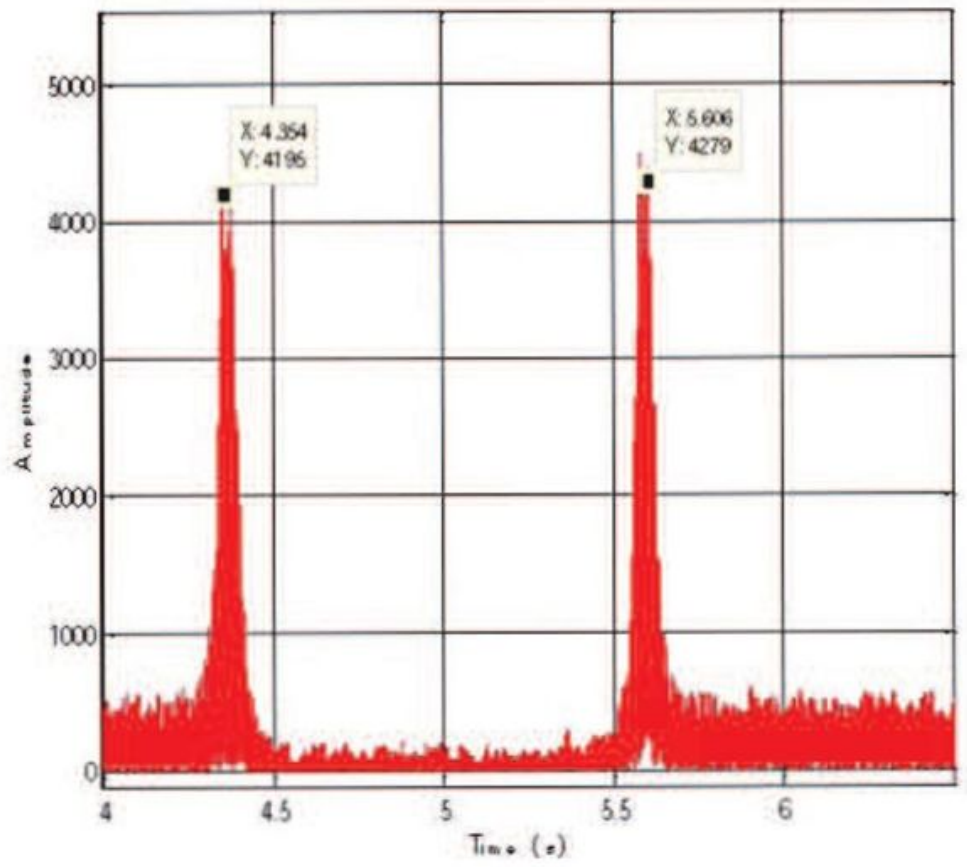

(b) $v=-10 \mathrm{~m} / \mathrm{s}$

Figure 6

The output of matched filtering under various $v$ 


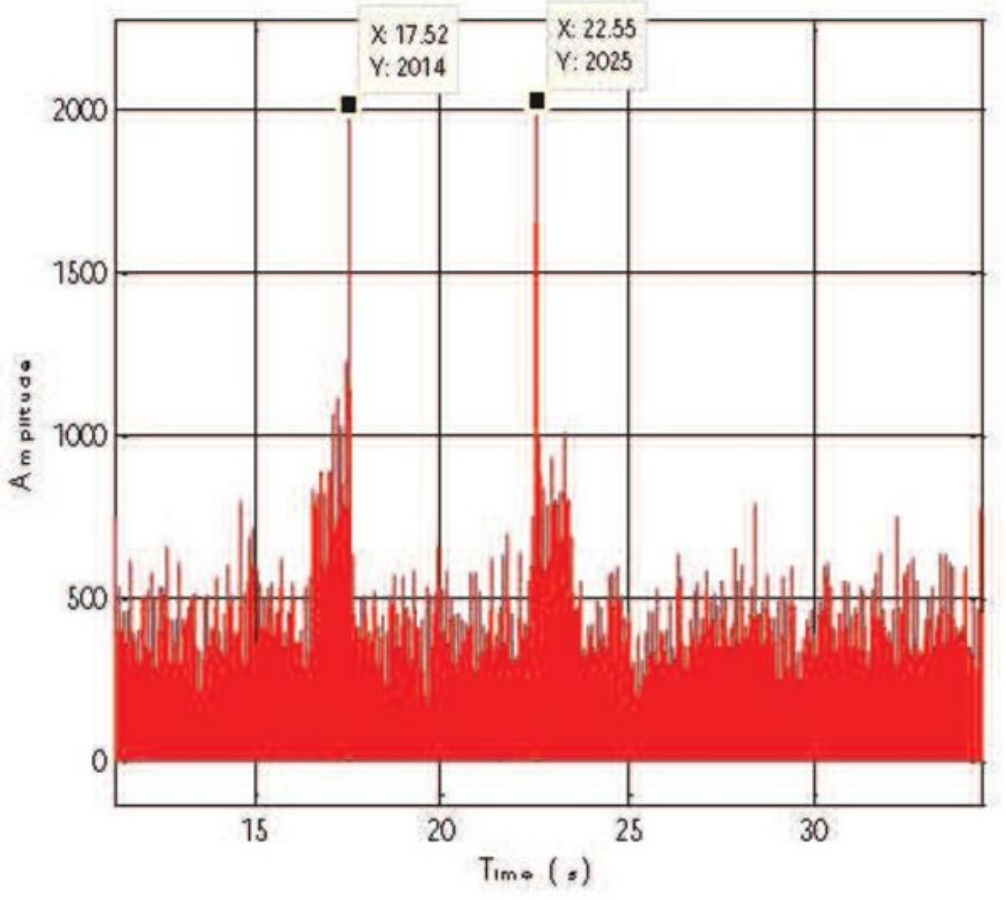

(a) $v=20 \mathrm{~m} / \mathrm{s}$

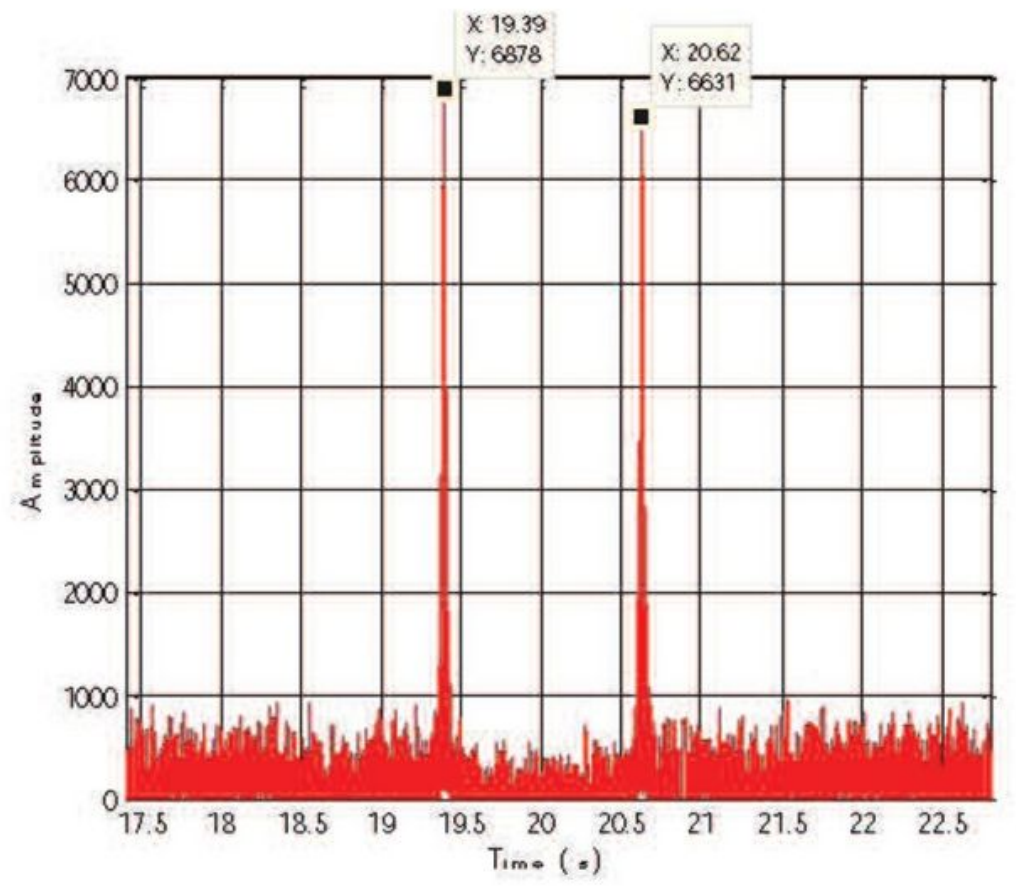

(b) $v=5 \mathrm{~m} / \mathrm{s}$

Figure 7

The output of matched filtering under various $v$ 